\title{
LHC sensitivity to lepton flavour violating $Z$ boson decays
}

\section{Sacha Davidson, Sylvain Lacroix and Patrice Verdier}

IPNL, Université de Lyon, Université Lyon 1, CNRS/IN2P3, 4 rue E. Fermi 69622 Villeurbanne cedex, France

E-mail: s.davidson@ipnl.in2p3.fr, s.lacroix@ipnl.in2p3.fr, verdier@ipnl.in2p3.fr

ABSTRACT: We estimate that the LHC could set bounds $B R\left(Z \rightarrow \mu^{ \pm} e^{\mp}\right)<4.1 \times 10^{-7}$ and $B R\left(Z \rightarrow \tau^{ \pm} \mu^{\mp}\right)<3.5 \times 10^{-6}$ (at $95 \%$ C.L.) with $20 \mathrm{fb}^{-1}$ of data at $8 \mathrm{TeV}$. A similar sensitivity can be anticipated for $Z \rightarrow \tau^{ \pm} e^{\mp}$, because we consider leptonic $\tau$ decays such that $Z \rightarrow \tau^{ \pm} \mu^{\mp} \rightarrow e^{ \pm} \mu^{\mp}+$ invisible. These limits can be compared to the LEP1 bounds of order $10^{-5} \rightarrow 10^{-6}$. Such collider searches are sensitive to a flavour-changing effective $Z$ coupling which is energy dependent, so are complementary to bounds obtained from $\tau \rightarrow \ell \bar{\ell} \ell$ and $\mu \rightarrow e \bar{e} e$.

Keywords: Rare Decays, Beyond Standard Model, Phenomenological Models

ARXIV EPRINT: 1207.4894 


\section{Contents}

1 Introduction 1

2 Theoretical review $\quad 2$

2.1 Flavour-changing $Z$ couplings 2

2.2 Kinematics 5

$\begin{array}{llr}3 & \text { Background and signal simulation } & 6\end{array}$

4 Sensitivity at the $8 \mathrm{TeV}$ LHC $\quad 7$

4.1 The $p p \rightarrow Z \rightarrow \mu^{ \pm} \tau^{\mp}$ channel $\quad 7$

4.2 The $p p \rightarrow Z \rightarrow e^{ \pm} \mu^{\mp}$ channel 9

$\begin{array}{lll}4.3 & \text { Limits } & 10\end{array}$

5 Summary 11

\section{Introduction}

Lepton flavour is conserved in the Standard Model (SM) of particle physics. However, the observation of neutrino oscillations implies that there is Beyond-the-Standard-Model physics which changes lepton flavour. Lepton Flavour Violation (LFV) among charged leptons is therefore a phenomenologically motivated place to search for elusive Beyondthe-Standard-Model (BSM) physics.

The LHC is an electroweak-scale hadron collider, so can reasonably be expected to give information on electroweak symmetry breaking, weakly interacting dark matter candidates (WIMPs) and new particles or interactions in the quark sector. In this paper, we are interested in the prospects of Lepton Flavour Violating processes at the LHC, involving SM particles. LFV in BSM scenarios, such as supersymmetry or heavy singlet neutrinos, has received more attention $[1-10]$.

The LHC produces many $\tau \mathrm{s}$ in the decays of $W$ and $Z$ bosons, and $b$ s (respectively $\sim 10^{8}, 3 \times 10^{7}$, and $10^{12}$ for $10 \mathrm{fb}^{-1}$ at $7 \mathrm{TeV}[11]$ ), so one could hope to be sensitive to LFV $\tau$ decays such as $\tau \rightarrow \ell \gamma$. LHCb obtained a bound $B R(\tau \rightarrow \mu \bar{\mu} \mu)<7.8 \times 10^{-8}$ with $1 \mathrm{fb}^{-1}$ of data ${ }^{1}[12]$. However, for ATLAS and CMS, there do not seem to be enough high- $p_{T} \tau \mathrm{s}$, to allow an interesting sensitivity $[11,13]$. We therefore explore LFV in the production of leptons - that is - in LFV decays of the $Z .^{2}$ The cross-section times branching ratio for

\footnotetext{
${ }^{1}$ To be compared with the Belle bound in table 2 .

${ }^{2} \mathrm{LFV}$ in the decay of Higgs produced at hadron colliders was discussed in $[14,15]$, taking into account the constraints from low energy processes. An earlier study of the collider prospects was [16]. This subject has recently been revisited [17].
} 
$p p \rightarrow Z \rightarrow \mu^{+} \mu^{-}$is of order a nanobarn at the 7 or $8 \mathrm{TeV}$ LHC, so with $20 \mathrm{fb}^{-1}$ of data at the end of 2012 , the LHC will have $2 \times 10^{7} / B R\left(Z \rightarrow \mu^{+} \mu^{-}\right) \sim 5 \times 10^{8} Z \mathrm{~s}$. This can be compared to the $1.7 \times 10^{7} Z \mathrm{~s}$ at LEP1. Effective LFV $Z$ couplings can also be constrained by the possible $Z$ contribution to decays such as $\tau \rightarrow \mu \bar{\mu} \mu$ and $\mu \rightarrow e \bar{e} e$. In section 2 , we review the complementarity of the collider and low energy searches.

We study the decays $Z \rightarrow \mu^{ \pm} e^{\mp}$ and $Z \rightarrow \tau^{ \pm} \mu^{\mp}$ (which results we extrapolate to $Z \rightarrow \tau^{ \pm} e^{\mp}$ ). We focus on leptonic $\tau$ decays, so the $\tau^{ \pm} \ell^{\mp}$ final state is $e^{ \pm} \mu^{\mp}+$ two neutrinos. This is not without backgrounds, as discussed in section 3. The most dangerous is $Z \rightarrow$ $\tau^{+} \tau^{-} \rightarrow e^{ \pm} \mu^{\mp}+4$ neutrinos, so section 2 also reviews some kinematic considerations, which suggest that this background could be reduced to an acceptable level. In section 4, we present our selection criteria, designed to maximise sensitivity to the signal. The bounds this analysis could obtain are in the final summary, which also discusses future prospects.

\section{Theoretical review}

The three $Z$ decays which violate lepton flavour conservation are $Z \rightarrow e^{ \pm} \mu^{\mp}, Z \rightarrow \mu^{ \pm} \tau^{\mp}$, and $Z \rightarrow e^{ \pm} \tau^{\mp}$. We consider the detection of $e^{ \pm} \mu^{\mp}$ final states, arising either directly, $Z \rightarrow$ $e^{ \pm} \mu^{\mp}$, or through leptonic tau decays: $Z \rightarrow \mu^{ \pm} \tau^{\mp} \rightarrow \mu^{ \pm} e^{\mp} \nu \bar{\nu}$, and $Z \rightarrow e^{ \pm} \tau^{\mp} \rightarrow e^{ \pm} \mu^{\mp} \nu \bar{\nu}$. As the last two decay channels differ only by exchange of the electron and the muon, we only consider $Z \rightarrow \mu^{ \pm} \tau^{\mp} \rightarrow \mu^{ \pm} e^{\mp} \nu \bar{\nu}$ in this paper. We anticipate that the sensitivity to $Z \rightarrow e^{ \pm} \tau^{\mp}$ should be equivalent, or better, because the SM backgrounds should be similar to those of $Z \rightarrow \mu^{ \pm} \tau^{\mp} \rightarrow \mu^{ \pm} e^{\mp} \nu \bar{\nu}$, and the detection prospects for a low $p_{T}$ muon (from tau decay) are better than those of a low $p_{T}$ electron.

\section{$2.1 \quad$ Flavour-changing $Z$ couplings}

We suppose that new LFV particles arise at a scale $M>m_{Z}$, which allows to describe the flavour changing $Z$ vertices with Effective Field Theory, via operators of dimension six and higher [18]..$^{3}$ The discussion applies to any flavour changing vertex, although we focus on the $Z \tau^{+} \mu^{-}$interaction to avoid flavour index sums. At dimension six, Buchmuller and Wyler [19] give the following SM gauge invariant operators which can contribute (with their hermitian conjugates) to $Z \rightarrow \tau^{ \pm} \mu^{\mp}$ :

$$
\begin{array}{lll}
{\left[H^{\dagger} \sigma^{I} D_{\alpha} H\right]\left[\bar{\ell}_{\mu} \sigma^{I} \gamma^{\alpha} \ell_{\tau}\right],} & {\left[H^{\dagger} D_{\alpha} H\right]\left[\bar{\ell}_{\mu} \gamma^{\alpha} \ell_{\tau}\right],} & {\left[H^{\dagger} D_{\alpha} H\right] \bar{\mu} \gamma^{\alpha} \tau} \\
\bar{\ell}_{\mu} \sigma^{I} \gamma_{\beta} D_{\alpha} \ell_{\tau} W^{I \alpha \beta}, & \bar{\ell}_{\mu} \gamma_{\beta} D_{\alpha} \ell_{\tau} B^{\alpha \beta}, & \bar{\mu} \gamma_{\beta} D_{\alpha} \tau B^{\alpha \beta} \\
\bar{\ell}_{\mu} \sigma^{I} H \sigma_{\beta \alpha} \tau W^{I \alpha \beta}, & \bar{\ell}_{\mu} H \sigma_{\beta \alpha} \tau B^{\alpha \beta} &
\end{array}
$$

where $\mathrm{SU}(2)$ contractions are indicated in square brackets when there is more than one possbility, $\ell_{\mu}$ is the muon doublet, $\tau$ the tau singlet, $B^{\alpha}$ the hypercharge gauge boson, $\sigma^{I}$ are the Pauli matrices, $\sigma_{\beta \alpha}=\frac{i}{2}\left[\gamma_{\beta}, \gamma_{\alpha}\right], B^{\alpha \beta}=\partial^{\alpha} B^{\beta}-\partial^{\beta} B^{\alpha}$ and so on. We suppose that these operators are normalised such that, in the presence of electroweak symmetry

\footnotetext{
${ }^{3}$ Our discussion is patterned on [18]; additional references can be found in the references and citations thereof.
} 
breaking, the combination of operators on the first (or second, third) line of equation (2.1)(2.3) appear in the Lagrangian as the first (or second, third) line below:

$$
\begin{aligned}
& g_{Z} m_{Z}^{2}\left[\bar{\mu} \gamma_{\alpha}\left(A_{L \mu \tau} P_{L}+A_{R \mu \tau} P_{R}\right) Z^{\alpha} \tau+h . c .\right] \\
& +2 g_{Z}\left[\bar{\mu} \gamma_{\alpha}\left(C_{L \mu \tau} P_{L}+C_{R \mu \tau} P_{R}\right) \partial^{\beta} \tau+h . c .\right] Z^{\beta \alpha} \\
& \quad+i g_{Z} m_{\tau}\left[\bar{\mu} \sigma_{\alpha \beta}\left(D_{L \mu \tau}^{Z} P_{L}+D_{R \mu \tau}^{Z} P_{R}\right) \tau-h . c .\right] Z^{\alpha \beta}
\end{aligned}
$$

where $g_{Z}=\sqrt{g^{2}+g^{\prime 2}}, Z^{\alpha \beta}=\partial^{\alpha} Z^{\beta}-\partial^{\beta} Z^{\alpha}$, and the $A, C, D$ coefficients have mass dimension -2 . We assume that these interactions are generated by a loop involving particles of mass $\sim M>m_{Z}$, so $A, C, D \propto g_{Z}^{2} /\left(16 \pi^{2} M^{2}\right)$. Concretely, if $C_{L \mu \tau}, C_{R \mu \tau}$ are taken real, and the $Z$ is on-shell, then equation (2.5) and the SM $Z$ couplings give

$$
\begin{aligned}
& \frac{-g}{2 \cos \theta_{W}}(\bar{\tau} \bar{\mu}) \gamma^{\alpha} Z_{\alpha} \times \\
& \quad \times\left[\begin{array}{cc}
g_{V}-g_{A} \gamma_{5} & m_{Z}^{2}\left[C_{L \mu \tau}+C_{R \mu \tau}-\left(C_{L \mu \tau}-C_{R \mu \tau}\right) \gamma_{5}\right] \\
m_{Z}^{2}\left[C_{L \mu \tau}+C_{R \mu \tau}-\left(C_{L \mu \tau}-C_{R \mu \tau}\right) \gamma_{5}\right] & g_{V}-g_{A} \gamma_{5}
\end{array}\right]\left(\begin{array}{l}
\tau \\
\mu
\end{array}\right)
\end{aligned}
$$

where $g_{V}=-1 / 2+2 s_{W}^{2}$ and $g_{A}=-1 / 2$.

The effective interactions relevant to LFV $Z$ decays are those of equation (2.5). This is because the $A$ and $D$ coefficients are constrained by low energy data (see below), whereas the coefficients $C_{L}, C_{R}$ are multiplied by the $Z$ four-momentum-squared, which suppresses their contribution in low-energy tree ${ }^{4}$ processes. For instance, the effective flavour-changing coupling on the $Z$ pole would be $g_{Z} m_{Z}^{2}\left(C_{L \mu \tau} P_{L}+C_{R \mu \tau} P_{R}\right)$, whereas in $\tau \rightarrow \mu \bar{\mu} \mu$ it would be $\lesssim g_{Z} m_{\tau}^{2}\left(C_{L \mu \tau} P_{L}+C_{R \mu \tau} P_{R}\right)$. Their contribution to $Z$ decays is:

$$
B R\left(Z \rightarrow \tau^{ \pm} \mu^{\mp}\right) \simeq 16 m_{Z}^{4}\left(\left|C_{L \mu \tau}\right|^{2}+\left|C_{R \mu \tau}\right|^{2}\right) \times B R\left(Z \rightarrow \mu^{ \pm} \mu^{\mp}\right) \sim 1.7 \times 10^{-5} \frac{m_{Z}^{4}}{M^{4}}
$$

where $g_{V} \rightarrow 0$ and the last estimate is for $C_{L \mu \tau} \simeq C_{R \mu \tau} \simeq g_{Z}^{2} /\left(16 \pi^{2} M^{2}\right)$. Recall that the effective operator formalism supposes $m_{Z}<M$, so constraints $\lesssim 10^{-5}$ are interesting. The current bounds on the $C$ s which can be extracted from the OPAL and DELPHI bounds on LFV $Z$ decays $[20,21]$ are given in table 1.

Bounds on the $C$ couplings can also be obtained from the contribution of a $Z$ loop to decays such as $\tau \rightarrow \mu \gamma$. To estimate a bound, we assume that $M \gg m_{Z}$, so the effective LFV vertices can be treated as contact interactions in the loop, and we suppose that at the scale $M$, New Physics induces $C \sim g_{Z}^{2} / 16 \pi^{2} M^{2}$ and no $A$ or $D$ coefficients. Then, renormalisation group mixing from the scale $M$ to $m_{Z}$ can induce $D^{\gamma}$ coefficients, which we estimate (for the case of $\tau \rightarrow \mu \gamma$ ) to be

$$
e m_{\tau} D_{\mu \tau}^{\gamma} \sim e m_{\tau} \frac{g_{Z}^{2} C_{\mu \tau}^{Z}}{32 \pi^{2}} \log \frac{m_{Z}^{2}}{M^{2}} \sim e m_{\tau}\left(\frac{g_{Z}^{2}}{16 \pi^{2}}\right)^{2} \frac{1}{2 M^{2}} \log \frac{m_{Z}^{2}}{M^{2}}
$$

The last colomn of table 2 gives bounds on the $D_{\alpha \beta}^{\gamma}$ s, which can translated into bounds on the $C_{\alpha \beta}^{Z}$ coefficients via equation (2.9). The $\mu \rightarrow e \gamma$ decay imposes $m_{Z}^{2} C_{e \mu}^{Z} \lesssim 10^{-5}$, which

\footnotetext{
${ }^{4}$ We thank Micheal Peskin for pointing out that this suppression may not occur in loops.
} 


\begin{tabular}{|c|c|c|}
\hline process & exp. limit & $16 \pi^{2} m_{Z}^{2} C / g_{Z}^{2}<$ \\
\hline$B R\left(Z \rightarrow e^{ \pm} \mu^{\mp}\right)(\mathrm{a})$ & $1.7 \times 10^{-6}$ & 0.32 \\
$B R\left(Z \rightarrow e^{ \pm} \tau^{\mp}\right)(\mathrm{a})$ & $9.8 \times 10^{-6}$ & 0.76 \\
$B R\left(Z \rightarrow \mu^{ \pm} \tau^{\mp}\right)(\mathrm{b})$ & $1.2 \times 10^{-5}$ & 0.84 \\
\hline
\end{tabular}

Table 1. Bounds on lepton flavour changing $Z$ decays (a) from OPAL [20] and (b) from DELPHI [21]. The third colomn assumes the decay $Z \rightarrow l_{\alpha}^{ \pm} l_{\beta}^{\mp}$ is mediated by $C_{L \alpha \beta} \simeq C_{R \alpha \beta} \simeq$ $g_{Z}^{2} / 16 \pi^{2} M^{2}$, and gives the resulting bound on $m_{Z}^{2} / M^{2}$ (which is different for the various $l_{\alpha}^{ \pm} l_{\beta}^{\mp}$ ).

corresponds to $B R\left(Z \rightarrow e \pm \mu^{\mp}\right) \lesssim 10^{-10}$. The $\tau \rightarrow \ell \gamma$ decays allow $M \sim m_{Z}$, so the loop calculation makes little sense, and the best bounds are the LEP limits of table 1.

We can make conflicting guesses for the "probable" values of $C_{L}$ and $C_{R}$. If the new particle mass scale $M$ is beyond the reach of the LHC, then so are the LFV $Z$ branching ratios (see equation 2.8). Also, as discussed below, the $A$ and $D$ coefficients are constrained by low energy rare decay data to be smaller than the LEPI bounds on the $C$ s. It is unclear to the authors how to build a model ${ }^{5}$ with $A, D \ll C$. However, the $C$ s are physically distinct, so it is phenomenologically interesting to constrain them. Furthermore, the OPAL experiment observed a $e^{+} e^{-} \rightarrow e^{+} \mu^{-}$event at LEP2 [22], which increases the interest of high centre-of-mass-energy searches for LFV.

To obtain bounds from processes at scales $\ll m_{Z}$, the $Z$ and its interactions (2.4)(2.6) can be matched onto LFV four fermion operators. Current rare lepton decay bounds constrain the coefficients $A_{L \mu \tau}, A_{R \mu \tau}$ more strictly ${ }^{6}$ than the $Z$ decay bounds of table 1 . The branching ratios mediated by the $A$ coefficients are

$$
\begin{aligned}
& \frac{B R\left(Z \rightarrow \tau^{ \pm} \mu^{\mp}\right)}{B R\left(Z \rightarrow \mu^{ \pm} \mu^{\mp}\right)} \simeq \frac{m_{Z}^{4}}{s_{W}^{4}}\left(\left|A_{L \mu \tau}\right|^{2}+\left|A_{R \mu \tau}\right|^{2}\right), \\
& \frac{B R(\tau \rightarrow \mu \bar{\mu} \mu)}{B R\left(\tau^{ \pm} \rightarrow \mu^{ \pm} \nu \bar{\nu}\right)}=\frac{m_{Z}^{4}}{8}\left(2\left|A_{L \mu \tau}\right|^{2}+\left|A_{R \mu \tau}\right|^{2}\right)
\end{aligned}
$$

so the rare lepton decay bounds of table 2 put $A_{L}$ and $A_{R}$ at or beyond the sensitivity of the 7 or $8 \mathrm{TeV}$ LHC.

We neglect the $D$ coefficients of equation (2.6), because their contribution to $B R(Z \rightarrow$ $\left.\tau^{ \pm} \mu^{\mp}\right)$ is proportional to $m_{\tau}^{4}$. This is different from the photon dipole operator

$$
i e m_{\tau}\left[\bar{\mu} \sigma_{\alpha \beta}\left(D_{L \mu \tau}^{\gamma} P_{L}+D_{R \mu \tau}^{\gamma} P_{R}\right) \tau-h . c .\right] F^{\alpha \beta}
$$

where the $m_{\tau}^{4}$ factor is cancelled in $B R(\tau \rightarrow \mu \gamma)$ by a similar factor in the denominator. The $\mathrm{SU}(2) \times \mathrm{U}(1)$ gauge invariant operators of equation (2.3) induce both $D^{\gamma}$ and $D^{Z}$,

\footnotetext{
${ }^{5} A$ could be suppressed with respect to $C$ if the loop particles do not couple to the Higgs, but this does not suppress $D$ couplings.

${ }^{6}$ The operators inducing $A$ coefficients also induce flavour-changing four fermion operators involving neutrinos. These are refered to as "Non-Standard neutrino Interactions", and can appear linearly in probabilities. Despite this potential enhanced sensitivity, the bounds [23] on $16 \pi^{2} A m_{Z}^{2} / g_{Z}^{2}$ are generically $\gtrsim 1$.
} 


\begin{tabular}{|c|c|c|c|}
\hline process & exp. limit & $16 \pi^{2} m_{Z}^{2} A / g_{Z}^{2}<$ & $16 \pi^{2} m_{Z}^{2} D / g_{Z}^{2}<$ \\
\hline$B R(\tau \rightarrow e \bar{e} e)$ & $2.7 \times 10^{-8}[24]$ & 0.17 & \\
$B R(\tau \rightarrow \mu \bar{\mu} \mu)$ & $2.1 \times 10^{-8}[24]$ & 0.15 & \\
$B R(\mu \rightarrow e \bar{e} e)$ & $1.0 \times 10^{-12}[25]$ & $4.3 \times 10^{-4}$ & \\
$B R(\tau \rightarrow e \gamma)$ & $3.3 \times 10^{-8}[26,27]$ & & $1.3 \times 10^{-3}$ \\
$B R(\tau \rightarrow \mu \gamma)$ & $4.4 \times 10^{-8}[26,27]$ & & $1.5 \times 10^{-3}$ \\
$B R(\mu \rightarrow e \gamma)$ & $2.4 \times 10^{-12}[28]$ & & $4.6 \times 10^{-6}$ \\
\hline
\end{tabular}

Table 2. Bounds from low energy Lepton Flavour Violating decays, on the $A$ and $D$ coefficients of equations (2.4) and (2.6). The third [fourth] colomn assumes the decay is mediated by $A_{L \mu \tau} \simeq$ $A_{R \mu \tau} \simeq g_{Z}^{2} / 16 \pi^{2} M^{2}\left[D_{L \mu \tau}^{\gamma} \simeq D_{R \mu \tau}^{\gamma} \simeq g_{Z}^{2} / 16 \pi^{2} M^{2}\right]$, and gives the bound on $m_{Z}^{2} / M^{2}$. If $e D^{\gamma} \sim$ $g_{Z} D^{Z}$, the $D^{\gamma}$ bounds would apply to $D^{Z}$.

so $D^{\gamma} \sim D^{Z}$ could be expected, in which case radiative lepton decays are more sensitive probes of dipole operators than $Z$ decays. In table 2 , are given the current bounds on $D_{L}^{\gamma}=D_{R}^{\gamma}$, normalised to $g_{Z}^{2} / 16 \pi^{2} m_{Z}^{2}$.

\subsection{Kinematics}

The LHC has more $Z$ s than LEP1, but a less clean environment. So we here review some simple kinematics, for the decays $Z \rightarrow \tau^{ \pm} \ell^{\mp}$, which suggest that the LHC backgrounds could be sufficiently reduced to probe couplings below the LEP1 bounds. The analysis for $Z \rightarrow \mu^{ \pm} e^{\mp}$ is relatively straightforward, since the $\mu^{ \pm} e^{\mp}$ should have the invariant mass of the $Z$, and is performed in section 4.2 .

In the case of $Z \rightarrow \tau^{ \pm} \ell^{\mp}$, we consider leptonic $\tau$ decays, in particular $\tau^{ \pm} \rightarrow e^{ \pm} \nu \bar{\nu}$, so as to obtain opposite flavour charged leptons in the final state:

$$
Z \rightarrow \tau^{ \pm} \mu^{\mp} \rightarrow\left(e^{ \pm} \nu \bar{\nu}\right) \mu^{\mp}
$$

The various backgrounds are studied in section 3 ; here we focus on the most problematic:

$$
Z \rightarrow \tau^{ \pm} \tau^{\mp} \rightarrow\left(e^{ \pm} \nu \bar{\nu}\right)\left(\mu^{\mp} \nu \bar{\nu}\right)
$$

which is refered to as "background" for the remainder of this section, and labelled $Z+$ jets in tables 3,5 and 7. To estimate the number of such events, recall that $B R\left(Z \rightarrow \tau^{ \pm} \tau^{\mp}\right)=$ 0.0337 and $B R\left(\tau^{ \pm} \rightarrow e^{ \pm} \nu \bar{\nu}\right)=0.178[29]$.

With some simple approximations, one can show that the two-neutrino signal events of equation (2.11) may be kinematically distinguishable from the four-neutrino background events. First, since the $\tau$ s are boosted by a factor of at least $m_{Z} / 2 m_{\tau}$, it is not unreasonable to approximate as collinear all the momenta of the $\tau$-daughters. For clarity, suppose that the $\tau^{+}$in the signal and background decays of equations (2.11) and (2.12) decays to $e^{+}$. Then in both decays:

$$
p_{\tau^{+}}=p_{e^{+}}+p_{\nu}+p_{\bar{\nu}} \equiv \alpha p_{e^{+}} .
$$


Similarly, for the $\tau^{-}$of the background process, one can define $p_{\tau^{-}}=\beta p_{\mu^{-}}$(where we neglect all final state masses). This gives:

$$
\begin{aligned}
m_{Z}^{2}-m_{\tau}^{2} & =2 \alpha p_{e^{+}} \cdot p_{\mu^{-}} & & \text {signal } \\
m_{Z}^{2}-2 m_{\tau}^{2} & =2 \alpha \beta p_{e^{+}} \cdot p_{\mu^{-}} & & \text {background }
\end{aligned}
$$

As expected, the invariant mass $2 p_{e^{+}} \cdot p_{\mu^{-}}$of the charged final state leptons should be lower for the background events, and the $p_{T}$ of the signal muon will have a harder distribution than that of the $\tau$ daughters (see figure 1).

The second approximation is to neglect the transverse momentum $p_{T, Z}$ of the $Z$, so that the $p_{T}$ of the $Z$-daughters balance. This implies that

$$
\begin{array}{llrl}
\alpha\left|p_{T, e^{+}}\right| & =\left|p_{T, \mu^{-}}\right| & & \text {signal } \\
\alpha\left|p_{T, e^{+}}\right| & =\beta\left|p_{T, \mu^{-}}\right| & & \text {background }
\end{array}
$$

To distinguish signal and background, we extract $\alpha_{1}$ and $\alpha_{2}$ from our "data" using respectively equations (2.14) and (2.16), and use the difference

$$
\Delta \alpha=\alpha_{2}-\alpha_{1}
$$

as a discriminating variable between background and signal (which peaks at zero).

There is a small fraction of background events, with $\beta \rightarrow 1$, which constitute an irreducible background to our signal. These correspond to events where the $\mu$ carries (almost) all the momentum of its parent- $\tau$. They will occur when, in the reference frame of the decaying $\tau$, the $\mu$ carries close to the maximum allowed energy $\left(m_{\tau}^{2}+m_{\mu}^{2}\right) / 2 m_{\tau}$, and when it is emitted in the direction of the boost of the $\tau$. If the charged lepton energies are measured with a fractional uncertainty $\epsilon$, then we estimate that such irreducible events correspond to $\sim \epsilon^{2}$ of the background. For $\epsilon \sim$ few $\%$, this suggests that the background of equation (2.12) can be sufficiently reduced.

\section{Background and signal simulation}

The signal investigated in this paper consists of events with exactly one electron and one muon. At the LHC, the dominant SM process leading to $e^{ \pm} \mu^{\mp}$ dilepton final states is $Z / \gamma^{*} \rightarrow \tau^{+} \tau^{-}$where both tau lepton decays leptonically, one to a muon and neutrinos and the other to an electron and neutrinos. Two processes involving top quark(s) also lead to $e^{ \pm} \mu^{\mp}$ final states: the top quark pair production channel $p p \rightarrow t \bar{t} \rightarrow b \ell^{+} \nu \bar{b} \ell^{\prime} \bar{\nu}$ and the single-top production channel $p p \rightarrow W t \rightarrow \ell^{ \pm} \nu b \ell^{\prime \mp} \nu$ with $\ell, \ell^{\prime}=e, \mu, \tau .^{7}$ Finally, gauge boson pair production can lead to $e^{ \pm} \mu^{\mp}$ states via: $p p \rightarrow W^{+} W^{-} \rightarrow \ell^{+} \nu \ell^{\prime} \bar{\nu}$ and $p p \rightarrow W Z / \gamma^{*} \rightarrow \ell^{\prime} \nu \ell^{+} \ell^{-}$with $\ell, \ell^{\prime}=e, \mu, \tau, p p \rightarrow W Z / \gamma^{*} \rightarrow q \bar{q}^{\prime} \tau^{+} \tau^{-}$, and $p p \rightarrow$ $Z / \gamma^{*} Z / \gamma^{*} \rightarrow f \bar{f} f^{\prime} \bar{f}^{\prime}$ with $f=q, \nu, e, \mu, \tau$.

The FEWZ [30] program was used to compute the inclusive $p p \rightarrow Z / \gamma^{*}$ cross section at next-to-next-to-leading order (NNLO) at $\sqrt{s}=8 \mathrm{TeV}$. The next-to-next-to-leading

\footnotetext{
${ }^{7}$ The notation $p p \rightarrow W t$ includes both $p p \rightarrow t W^{-}$and $p p \rightarrow \bar{t} W^{+}$.
} 
logarithm (NNLL) cross sections were taken from ref. [31] for $p p \rightarrow t \bar{t}$, and from ref. [32] for $p p \rightarrow t W$, with a top quark mass of $173.3 \mathrm{GeV}$. Finally, the diboson $(p p \rightarrow W W, W Z, Z Z)$ cross sections were computed using MCFM [33] at next-to-leading order (NLO). These results are used to predict the total number of background events, which we then simulate at leading order.

The simulation of those SM backgrounds and of the signal processes $p p \rightarrow Z \rightarrow$ $\mu^{ \pm} \tau^{\mp} \rightarrow \mu^{ \pm} e^{\mp} \nu \bar{\nu}$ and $p p \rightarrow Z \rightarrow e^{ \pm} \mu^{\mp}$ was performed using PYTHIA version 8.162 [34], except the simulation of the $p p \rightarrow W t \rightarrow \ell^{ \pm} \nu b \ell^{\prime \mp} \nu$ process which also used MADGRAPH5 version 1.4.6 [35]. In all Monte Carlo simulations, the CTEQ6L1 [36, 37] parton density functions were used; initial and final state radiations and multi-parton interactions were also included. However, pile-up effects due to additional interactions were not taken into account in those simulations.

All Monte Carlo events were passed through the DELPHES [38] program which provides a fast simulation of LHC detector response. The default configuration corresponding to the CMS detector [39] at the LHC was used. In the following, the pseudorapidity is defined as $\eta=-\ln [\tan (\theta / 2)]$ with $\theta$ the polar angle with respect to the beam direction; and $\phi$ is the azimuthal angle. Isolated electrons and muons were identified by requiring no track with $p_{T}>2 \mathrm{GeV}$ in a cone of radius $R=0.5$ around those lepton candidates, with $\Delta R=\sqrt{\Delta \eta^{2}+\Delta \phi^{2}}$. Standard Model backgrounds with non-isolated leptons were therefore not taken into account. Jets were reconstructed with the FASTJET [40] program using the anti-kt algorithm with a distance parameter of 0.7 .

Table 3 contains the details of the Monte Carlo event production performed for the studies reported in this paper. As a total integrated luminosity of $20 \mathrm{fb}^{-1}$ at $\sqrt{\mathrm{s}}=8 \mathrm{TeV}$ is assumed in the following analyses, the equivalent luminosities of these simulations are always higher than $200 \mathrm{fb}^{-1}$ for each process to reduce statistical uncertainties.

\section{Sensitivity at the $8 \mathrm{TeV}$ LHC}

For the signal investigated in this study, at least one lepton comes directly from the $Z$ boson decay. As shown in figure 1, the distribution of this lepton $p_{T}$ has a clear peak around $\sim 40 \mathrm{GeV}$, as expected for this jacobian distribution. At the LHC, a large fraction of SM backgrounds leading to $e^{ \pm} \mu^{\mp}$ final states comes $W$ and $Z$ bosons decaying to tau lepton(s) which decay leptonically $(\tau \rightarrow \ell \bar{\nu} \nu)$. In that case, the $p_{T}$ of the final lepton is strongly reduced due to the energy carried away by neutrinos (see figure 1). Requiring at least one high $p_{T}$ lepton, can significantly increase the signal to background ratio. This selection criterium also guarantees that events are accepted by single lepton trigger conditions of LHC experiments. In the following, 100\% trigger efficiency is therefore assumed.

\subsection{The $p p \rightarrow Z \rightarrow \mu^{ \pm} \tau^{\mp}$ channel}

In the $p p \rightarrow Z \rightarrow \mu^{ \pm} \tau^{\mp} \rightarrow \mu^{ \pm} e^{\mp} \bar{\nu} \nu$ channel, the muon comes directly from the $Z$ boson decay. The muon $p_{T}$ is required to be above $30 \mathrm{GeV}$ and in the detector pseudorapidity acceptance $|\eta|<2.1$. As the electron comes from the tau decay, the selection criterium on the electron $p_{T}$ has to be reduced to keep high signal efficiency. The electron $p_{T}$ is therefore 


\begin{tabular}{|c|l|r|r|}
\hline \multicolumn{2}{|c|}{ Processes } & $\sigma \times B R(\mathrm{pb})$ & $\begin{array}{r}\text { Number of } \\
\text { simulated events }\end{array}$ \\
\hline$Z$ s+jets & $\begin{array}{c}Z / \gamma^{*} \rightarrow \tau^{ \pm} \tau^{\mp} \rightarrow \ell^{ \pm} \nu \bar{\nu} \ell^{\prime \mp} \nu \bar{\nu} \\
\text { with } \ell, \ell^{\prime}=e, \mu\left(M_{Z / \gamma^{*}}>20 \mathrm{GeV}\right)\end{array}$ & 237. & $47,500,000$ \\
& \multicolumn{1}{|c|}{$t \bar{t} \rightarrow b \ell^{+} \nu \bar{b} \ell^{\prime-} \bar{\nu}$ with $\ell, \ell^{\prime}=e, \mu, \tau$} & 23.6 & $4,720,000$ \\
\hline single-top & $W t \rightarrow \ell^{ \pm} \nu b \ell^{\prime \mp} \nu$ with $\ell, \ell^{\prime}=e, \mu, \tau$ & 2.35 & 400,000 \\
\hline diboson & $W^{+} W^{-} \rightarrow \ell^{+} \nu \ell^{\prime-} \bar{\nu}$ with $\ell, \ell^{\prime}=e, \mu, \tau$ & 6.00 & $1,200,000$ \\
& $W Z / \gamma^{*} \rightarrow q \bar{q}^{\prime} \tau^{ \pm} \tau^{\mp}$ & 0.735 & 140,000 \\
& $W Z / \gamma^{*} \rightarrow \ell^{ \pm} \nu \ell^{+} \ell^{-}$with $\ell, \ell^{\prime}=e, \mu, \tau$ & 1.06 & 220,000 \\
& $Z / \gamma^{*} Z / \gamma^{*} \rightarrow f \bar{f} f^{\prime} \bar{f}^{\prime}$ with $f=q, \nu, e, \mu, \tau$ & 8.26 & $1,660,000$ \\
\hline signals & $Z \rightarrow \tau^{ \pm} \mu^{\mp} \rightarrow e^{ \pm} \nu \bar{\nu} \mu^{\mp}$ & & 100,000 \\
& $Z \rightarrow \mu^{ \pm} e^{\mp}$ & & 100,000 \\
\hline
\end{tabular}

Table 3. Standard model backgrounds in the search for $Z$ decay violating lepton number at the LHC; the second column contains $\sigma \times B R$ (in pb) for p-p collisions at $\sqrt{s}=8 \mathrm{TeV}$, and the third column shows the number of simulated events in this analysis which is in all cases greater than 10 times $\mathcal{L} \sigma B R$ with $\mathcal{L}=20 \mathrm{fb}^{-1}$.

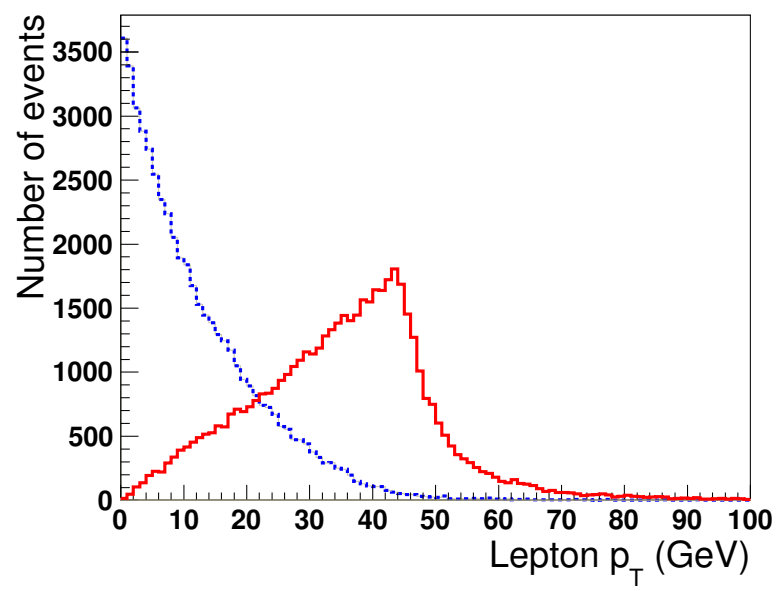

Figure 1. Lepton (electron or muon) $p_{T}$ distribution at generator level at the $8 \mathrm{TeV}$ LHC: for leptons from $p p \rightarrow Z / \gamma^{*} \rightarrow \ell^{+} \ell^{-}$(plain line) and for leptons from $p p \rightarrow Z \gamma^{*} \rightarrow \tau^{+} \tau^{-} \rightarrow \ell^{+} \nu \bar{\nu} \ell^{-} \bar{\nu} \nu$ (dotted line), with $\ell=e$ or $\mu$.

required to be above $10 \mathrm{GeV}$ and with $|\eta|<2.5$. To reduce the diboson background coming from events with more than 3 leptons, events are required to contain exactly 2 leptons. At this stage, a reducible diboson background comes from events where lepton(s) failed identification criteria leading to same-sign $e^{ \pm} \mu^{ \pm}$final states. This background is therefore rejected by requiring two opposite-sign leptons. Double and single-top quark production are then strongly reduced by rejecting events which contain at least one jet with $p_{T}>30 \mathrm{GeV}$ and $|\eta|<2.5$. To reduce the remaining diboson backgrounds, angular criteria in the 


\begin{tabular}{|l|r|r|}
\hline Selection criteria & $N_{\text {backgrd. }}$ & Signal efficiency (\%) \\
\hline$\geq 1$ muon with $p_{T}>30 \mathrm{GeV}$ and $|\eta|<2.1$ and & $48,181 \pm 68$ & $9.4 \pm 0.1$ \\
$\geq 1$ electron with $p_{T}>10 \mathrm{GeV}$ and $|\eta|<2.5$ & & \\
\hline exactly 2 leptons & $43,496 \pm 65$ & $9.4 \pm 0.1$ \\
2 leptons with opposite charge & $42,652 \pm 64$ & $9.4 \pm 0.1$ \\
jet veto: no jet with $p_{T}>30 \mathrm{GeV}$ and $|\eta|<2.5$ & $11,358 \pm 34$ & $7.8 \pm 0.1$ \\
$\Delta \phi(e, \mu)>2.7$ & $6,850 \pm 26$ & $6.9 \pm 0.1$ \\
$\Delta \phi\left(e, E_{T}\right)<0.7$ & $3,763 \pm 19$ & $6.2 \pm 0.1$ \\
$38 \mathrm{GeV}<M_{e \mu}<92 \mathrm{GeV}$ & $3,201 \pm 18$ & $6.1 \pm 0.1$ \\
\hline
\end{tabular}

Table 4. Selection criteria for the $Z \rightarrow \tau^{ \pm} \mu^{\mp}$ search at the $\sqrt{s}=8 \mathrm{TeV}$ LHC with $\mathcal{L}=20 \mathrm{fb} b^{-1}$ with the total number of events expected from SM backgrounds and the signal efficiency (\%); uncertainties are statistical only.

transverse plane are used. For the signal, electrons and muons are almost back-to-back in the transverse plane, while the distribution of the $\Delta \phi$ angle between the muon and the electron is more flat for the diboson backgrounds. This $\Delta \phi(e, \mu)$ angle is therefore required to be higher than 2.7 radians. Another difference between backgrounds and signal is the $\Delta \phi$ angle between the electron and the direction of the missing transverse energy $\left(E_{T}\right)$, which is peaked at 0 for signal since electrons and missing transverse energy both come from a higly boosted tau lepton. This $\Delta \phi\left(e, \mathbb{E}_{T}\right)$ angle is therefore required to be below 0.7 radians. Finally, the $e \mu$ invariant mass is required to be in the range [38 - 92] GeV, i.e. in the region around the broad peak expected for the signal. The numbers of events expected from SM backgrounds at each step of the event selection are reported in table 4, together with the signal efficiency.

The remaining SM backgrounds is 3201 events. As expected, 95\% of this background comes from the $Z / \gamma^{*} \rightarrow \tau^{ \pm} \tau^{\mp} \rightarrow \mu^{ \pm} e^{\mp} \nu \bar{\nu}$ background (see table 5). The signal efficiency is $6.1 \%$. Normalized to the $p p \rightarrow Z$ cross section multiplied by $B R\left(Z \rightarrow \tau^{ \pm} \mu^{ \pm}\right)=1.2 \times 10^{-5}$, which corresponds to the current $95 \%$ C.L. limit from LEP, the number of events expected for the signal is 489 .

\subsection{The $p p \rightarrow Z \rightarrow e^{ \pm} \mu^{\mp}$ channel}

In the $p p \rightarrow Z \rightarrow e^{ \pm} \mu^{\mp}$ channel, selection criteria are very close to those reported in previous section. The fact that this signal contains two high $p_{T}$ leptons with a narrow invariant mass allows to reach a much better signal to background ratio. The muon and the electron $p_{T}$ are required to be above $30 \mathrm{GeV}$ and their pseudorapidity $|\eta|<2.1$ and $<2.5$, respectively. Events are required to contain exactly two leptons, with opposite charge. The jet veto rejecting events with at least one jet with $p_{T}>30 \mathrm{GeV}$ and $|\eta|<2.5$ is then applied. Finally, the $\Delta \phi(e, \mu)$ angle is required to be higher than 2.7 radians. The numbers of events expected from SM backgrounds at each step of the event selection are reported in table 6 , together with the signal efficiency. 


\begin{tabular}{|l|r|}
\hline Processes & Number of events \\
\hline$Z+$ jets & $3,037 \pm 17$ \\
$t \bar{t}$ & $9 \pm 1$ \\
single-top & $7 \pm 1$ \\
diboson & $147 \pm 4$ \\
\hline Total SM backgrounds & $3,201 \pm 18$ \\
\hline$Z \rightarrow \tau^{ \pm} \mu^{\mp}$ signal & $489 \pm 6$ \\
\hline
\end{tabular}

Table 5. After selection criteria of table 4 for the $Z \rightarrow \tau^{ \pm} \mu^{\mp}$ search at the $\sqrt{s}=8 \mathrm{TeV}$ LHC with $\mathcal{L}=20 \mathrm{fb}^{-1}$, number of events expected for each SM background and for the signal normalized to $\sigma(p p \rightarrow Z) \times B R\left(Z \rightarrow \tau^{ \pm} \mu^{ \pm}\right)$with $B R\left(Z \rightarrow \tau^{ \pm} \mu^{ \pm}\right)=1.2 \times 10^{-5}$. These numbers correspond to figure 2 .

\begin{tabular}{|l|r|r|}
\hline Selection criteria & $N_{\text {backgrd. }}$ & Signal efficiency (\%) \\
\hline$\geq 1$ muon with $p_{T}>30 \mathrm{GeV}$ and $|\eta|<2.1$ and & $36,489 \pm 59$ & $18.5 \pm 0.1$ \\
$\geq 1$ electron with $p_{T}>30 \mathrm{GeV}$ and $|\eta|<2.5$ & & \\
\hline exactly 2 leptons & $34,787 \pm 58$ & $18.5 \pm 0.1$ \\
2 leptons with opposite charge & $34,038 \pm 58$ & $18.5 \pm 0.1$ \\
jet veto: no jet with $p_{T}>30 \mathrm{GeV}$ and $|\eta|<2.5$ & $8,111 \pm 28$ & $15.9 \pm 0.1$ \\
$\Delta \phi(e, \mu)>2.7$ & $4,771 \pm 22$ & $14.0 \pm 0.1$ \\
\hline
\end{tabular}

Table 6. Selection criteria for the $Z \rightarrow e^{ \pm} \mu^{\mp}$ search at the $\sqrt{s}=8 \mathrm{TeV}$ LHC with $\mathcal{L}=20 f b^{-1}$ with the total number of events expected from SM backgrounds and the signal efficiency (\%); uncertainties are statistical only.

The remaining SM backgrounds is 4771 events. At this stage the dominant background comes from $Z / \gamma^{*} \rightarrow \tau^{ \pm} \tau^{\mp} \rightarrow \mu^{ \pm} e^{\mp} \nu \bar{\nu}$ events (see table 7 ). And the signal efficiency is $14.0 \%$. Normalized to the $p p \rightarrow Z$ cross section multiplied by $B R\left(Z \rightarrow e^{ \pm} \mu^{ \pm}\right)=1.7 \times 10^{-6}$, which corresponds to the current $95 \%$ C.L. limit from LEP, the number of events expected for the signal is 158. In the mass region [87-95] around the $Z$ peak, this signal would give 121 events, whereas the total SM background represents 222 events, half of it coming $Z / \gamma^{*} \rightarrow \tau^{ \pm} \tau^{\mp} \rightarrow \mu^{ \pm} e^{\mp} \nu \bar{\nu}$ events and the other half from diboson events.

\subsection{Limits}

From the number of background events and the signal efficiencies reported in the previous section, expected $95 \%$ C.L. limits on the number of signal events can be computed and translated into limits on lepton flavour violating $Z$ branching ratios. The modified frequentist $C L_{s}[41,42]$ method was used to compute those expected limits. The shapes of the $\Delta \alpha$ (defined in equation 2.18) and $e^{ \pm} \mu^{\mp}$ invariant mass distributions were used as final discriminant variables in the limit computation for the $Z \rightarrow \tau^{ \pm} \mu^{\mp}$ and $Z \rightarrow e^{ \pm} \mu^{\mp}$ channel, respectively. Those distributions are shown in figure 2. A $3 \%$ systematic uncertainty was set on the SM background contributions and on the signal efficiencies. This uncertainty 


\begin{tabular}{|l|r|}
\hline Processes & Number of events \\
\hline$Z+$ jets & $2,756 \pm 17$ \\
$t \bar{t}$ & $199 \pm 4$ \\
single-top & $132 \pm 4$ \\
diboson & $1,683 \pm 13$ \\
\hline Total SM backgrounds & $4,771 \pm 22$ \\
\hline$Z \rightarrow e^{ \pm} \mu^{\mp}$ signal & $158 \pm 1$ \\
\hline
\end{tabular}

Table 7. After selection criteria of the $Z \rightarrow e^{ \pm} \mu^{\mp}$ search at the $\sqrt{s}=8 \mathrm{TeV}$ LHC with $\mathcal{L}=$ $20 \mathrm{fb}^{-1}$, number of events expected for each SM background and for the signal normalized to $\sigma(p p \rightarrow Z) \times B R\left(Z \rightarrow e^{ \pm} \mu^{ \pm}\right)$with $B R\left(Z \rightarrow e^{ \pm} \mu^{ \pm}\right)=1.7 \times 10^{-6}$. These numbers correspond to figure 2 .
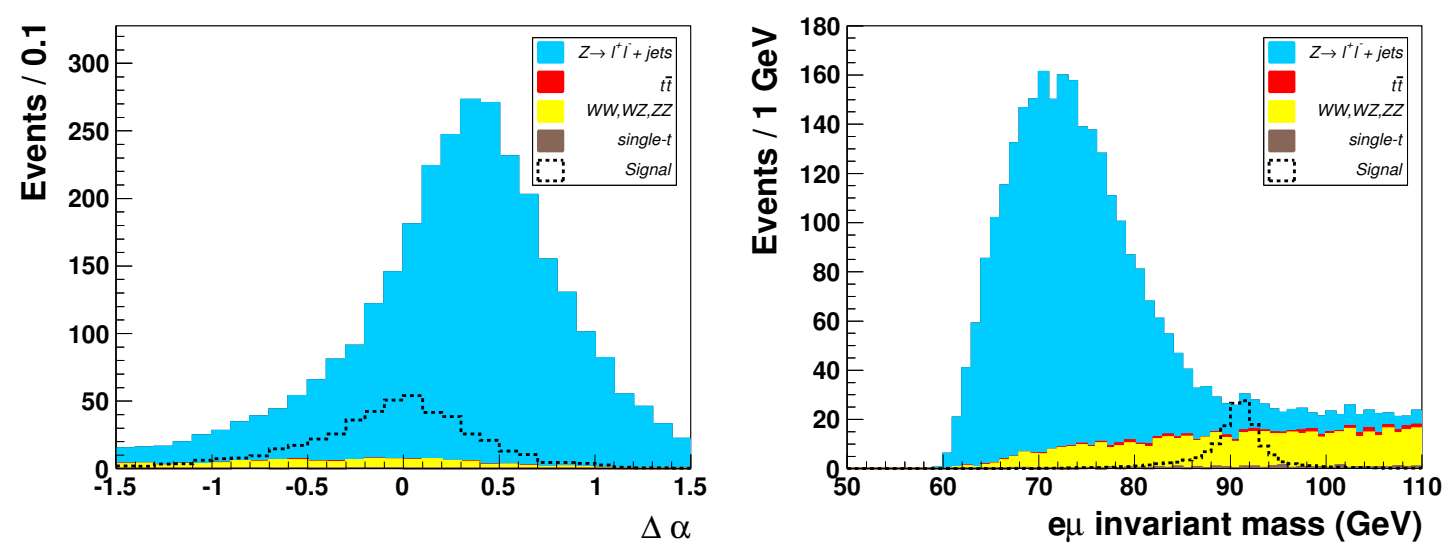

Figure 2. Search for flavour violating $\mathrm{Z}$ decay at the $\sqrt{s}=8 \mathrm{TeV}$ LHC with $\mathcal{L}=20 \mathrm{fb} b^{-1}$ : in the $Z \rightarrow \mu^{ \pm} \tau^{\mp} \rightarrow \mu^{ \pm} e^{\mp} \nu \bar{\nu}$, distribution of $\Delta \alpha$ after the final step of the event selection (left), and in the $Z \rightarrow e^{ \pm} \mu^{ \pm}$channel, distribution of the $e^{ \pm} \mu^{ \pm}$invariant mass after the final step of the event selection (right). The dashed signal corresponding to the LEP1 limits is superposed on the summed backgrounds.

accounts for the experimental uncertainty on lepton identification [43, 44], assuming that the uncertainty on luminosity measurement can be cancelled in our analysis using normalization to $p p \rightarrow Z \rightarrow \mu^{+} \mu^{-}$and $p p \rightarrow Z \rightarrow e^{+} e^{-}$events. This $3 \%$ systematic uncertainty was treated as fully correlated between signal and SM backgrounds. This procedure allows to set $95 \%$ C.L. expected limits of $3.5 \times 10^{-6}$ and $4.1 \times 10^{-7}$ on the $Z$ boson branching ratios $B R\left(Z \rightarrow \tau^{ \pm} \mu^{\mp}\right)$ and $B R\left(Z \rightarrow e^{ \pm} \mu^{\mp}\right)$, respectively. Those expected limits are a factor $\sim 4$ better than the current experimental limits from LEP.

\section{Summary}

The high number of $Z$ bosons produced at the LHC allows to study rare $Z$ boson decay predicted by BSM theories. Using $20 \mathrm{fb}^{-1}$ of $p p$ collisions at $8 \mathrm{TeV}$, we have shown that $95 \%$ C.L. limits on $Z$ decay branching ratios to $\tau^{ \pm} \mu^{\mp}$ and $e^{ \pm} \mu^{\mp}$ of $3.5 \times 10^{-6}$ and $4.1 \times 10^{-7}$, 
respectively, could be obtained. This represents a factor 4 improvements with respect to current best limits from LEP. The expected limit on $B R\left(Z \rightarrow \tau^{ \pm} e^{\mp}\right)$ should be equivalent to the one on $B R\left(Z \rightarrow \tau^{ \pm} \mu^{\mp}\right)$. As discussed in section 2 , such collider searches are generically complementary to rare lepton decay searches, because they are sensitive to an effective vertex $\propto s$, which gives small contributions to tree level low energy processes. As shown in eq. 2.8, reaching sensitivity to lower $B R$ s would be interesting to test BSM models. However, an effective $Z$ vertex $\propto s$ can also contribute in loops above $m_{Z}$; the limits on $\mu \rightarrow e \gamma$ are sufficiently restrictive to constrain this effect, implying that $B R\left(Z \rightarrow \mu^{ \pm} e^{\mp}\right) \lesssim 10^{-10}$.

In the analyses presented in this paper, basic and simple selection criteria were used to distinguish signals from SM backgrounds. More sophisticated analyses might further reduce backgrounds and increase sensitivities. In the limit computation, a 3\% total systematic uncertainy on signal and background has been assigned, assuming some systematics, as the one on the integrated luminosity, could be cancelled in this measurement.

It is interesting to speculate about the prospects of LFV $Z$ decays at the post-2014 LHC. Our analysis is not immediately applicable, as the relative importance of the backgrounds will vary with the increased centre-of-mass energy. However, for the analysis described here, the expected limits reduce by factors of 2 or 3 with a luminosity of $200 \mathrm{fb}^{-1}$, then further improvements are limited by the systematic uncertainties. Selection criteria which more effectively suppress the backgrounds, and reduced systematic uncertainties, would be required to profit from the full integrated luminosity of the future LHC.

\section{Acknowledgments}

We thank Gerald Grenier and Michael Peskin for useful suggestions.

Open Access. This article is distributed under the terms of the Creative Commons Attribution License which permits any use, distribution and reproduction in any medium, provided the original author(s) and source are credited.

\section{References}

[1] F. del Aguila, J. Aguilar-Saavedra, B. Allanach, J. Alwall, Y. Andreev, et al., Collider aspects of flavour physics at high Q, Eur. Phys. J. C 57 (2008) 183 [arXiv:0801.1800] [InSPIRE].

[2] I. Hinchliffe and F. Paige, Lepton flavor violation at the CERN LHC, Phys. Rev. D 63 (2001) 115006 [hep-ph/0010086] [INSPIRE].

[3] R. Allahverdi, B. Dutta, T. Kamon and A. Krislock, Lepton flavor violation at the Large Hadron Collider, Phys. Rev. D 86 (2012) 015026 [arXiv:1203.3276] [INSPIRE].

[4] K. Hamaguchi and A. Ibarra, Probing lepton flavor violation in slepton NLSP scenarios, JHEP 02 (2005) 028 [hep-ph/0412229] [INSPIRE].

[5] S. Kaneko, J. Sato, T. Shimomura, O. Vives and M. Yamanaka, Measuring lepton flavour violation at LHC with long-lived slepton in the coannihilation region, Phys. Rev. D 78 (2008) 116013 [arXiv:0811.0703] [INSPIRE]. 
[6] A. Bartl et al., Test of lepton flavor violation at LHC, Eur. Phys. J. C 46 (2006) 783 [hep-ph/0510074] [INSPIRE].

[7] ATLAS collaboration, G. Aad et al., Search for lepton flavour violation in the emu continuum with the ATLAS detector in $\sqrt{s}=7 \mathrm{TeV}$ pp collisions at the LHC, Eur. Phys. J. C 72 (2012) 2040 [arXiv:1205.0725] [INSPIRE].

[8] D0 collaboration, V.M. Abazov et al., Search for sneutrino production in emu final states in $5.3 \mathrm{fb}^{-1}$ of $p \bar{p}$ collisions at $\sqrt{s}=1.96 \mathrm{TeV}$, Phys. Rev. Lett. 105 (2010) 191802 [arXiv: 1007.4835$]$ [INSPIRE].

[9] F. del Aguila and J. Aguilar-Saavedra, Distinguishing seesaw models at LHC with multi-lepton signals, Nucl. Phys. B 813 (2009) 22 [arXiv:0808.2468] [INSPIRE].

[10] A. Akeroyd, C.-W. Chiang and N. Gaur, Leptonic signatures of doubly charged Higgs boson production at the LHC, JHEP 11 (2010) 005 [arXiv: 1009.2780] [INSPIRE].

[11] CMS collaboration, M Giffels et al., Lepton flavour violation in the neutrinoless $\tau$ decay $\tau \rightarrow 3 \mu$ with the CMS experiment, CMS-CR-2009-013 (2009).

[12] LHCb collaboration, I. Bediaga et al., Implications of LHCb measurements and future prospects, [arXiv: 1208.3355] [INSPIRE].

[13] ATLAS collaboration, L. Serin and R. Stroynowski, Study of lepton number violating decay $\tau \rightarrow \mu \gamma$ in ATLAS, ATL-PHYS-97-114 (1997).

[14] S. Kanemura, T. Ota and K. Tsumura, Lepton flavor violation in Higgs boson decays under the rare $\tau$ decay results, Phys. Rev. D 73 (2006) 016006 [hep-ph/0505191] [INSPIRE].

[15] S. Davidson and G.J. Grenier, Lepton flavour violating Higgs and tau to mu gamma, Phys. Rev. D 81 (2010) 095016 [arXiv: 1001. 0434] [INSPIRE].

[16] K.A. Assamagan, A. Deandrea and P.-A. Delsart, Search for the lepton flavor violating decay A0/H0 $\rightarrow \tau^{ \pm} \mu^{\mp}$ at hadron colliders, Phys. Rev. D 67 (2003) 035001 [hep-ph/0207302] [INSPIRE].

[17] A. Goudelis, O. Lebedev and J.-h. Park, Higgs-induced lepton flavor violation, Phys. Lett. B 707 (2012) 369 [arXiv:1111.1715] [INSPIRE].

[18] A. Brignole and A. Rossi, Anatomy and phenomenology of $\mu-\tau$ lepton flavor violation in the MSSM, Nucl. Phys. B 701 (2004) 3 [hep-ph/0404211] [INSPIRE].

[19] W. Buchmüller and D. Wyler, Effective lagrangian analysis of new interactions and flavor conservation, Nucl. Phys. B 268 (1986) 621 [INSPIRE].

[20] OPAL collaboration, R. Akers et al., A search for lepton flavor violating $Z_{0}$ decays, Z. Phys. C 67 (1995) 555 [inSPIRE].

[21] DELPHI collaboration, P. Abreu et al., Search for lepton flavor number violating $Z_{0}$ decays, Z. Phys. C 73 (1997) 243 [inSPIRE].

[22] OPAL collaboration, G. Abbiendi et al., Search for lepton flavor violation in $e^{+} e^{-}$collisions at $\sqrt{s}=189 \mathrm{GeV}-209 \mathrm{GeV}$, Phys. Lett. B 519 (2001) 23 [hep-ex/0109011] [INSPIRE].

[23] C. Biggio, M. Blennow and E. Fernandez-Martinez, General bounds on non-standard neutrino interactions, JHEP 08 (2009) 090 [arXiv:0907.0097] [INSPIRE].

[24] K. Hayasaka et al., Search for lepton flavor violating $\tau$ decays into three leptons with 719 million produced $\tau^{+} \tau^{-}$pairs, Phys. Lett. B 687 (2010) 139 [arXiv:1001.3221] [INSPIRE].

[25] SINDRUM collaboration, U. Bellgardt et al., Search for the decay $\mu^{+} \rightarrow e^{+} e^{+} e^{-}$, Nucl. Phys. B 299 (1988) 1 [INSPIRE]. 
[26] BABAR collaboration, B. Aubert et al., Searches for lepton flavor violation in the decays $\tau^{ \pm} \rightarrow e^{ \pm} \gamma$ and $\tau^{ \pm} \rightarrow \mu^{ \pm} \gamma$, Phys. Rev. Lett. 104 (2010) 021802 [arXiv:0908.2381] [INSPIRE].

[27] Belle collaboration, K. Hayasaka et al., New search for $\tau \rightarrow \mu \gamma$ and $\tau \rightarrow$ e $\gamma$ decays at Belle, Phys. Lett. B 666 (2008) 16 [arXiv:0705.0650] [INSPIRE].

[28] MEG collaboration, J. Adam et al., New limit on the lepton-flavour violating decay $\mu^{+} \rightarrow e^{+} \gamma$, Phys. Rev. Lett. 107 (2011) 171801 [arXiv:1107.5547] [INSPIRE].

[29] Particle Data Group collaboration, J. Beringer et al., Review of particle physics, Phys. Rev. D 86 (2012) 010001 [inSPIRE].

[30] R. Gavin, Y. Li, F. Petriello and S. Quackenbush, FEWZ 2.0: a code for hadronic Z production at next-to-next-to-leading order, Comput. Phys. Commun. 182 (2011) 2388 [arXiv: 1011.3540] [INSPIRE].

[31] M. Cacciari, M. Czakon, M. Mangano, A. Mitov and P. Nason, Top-pair production at hadron colliders with next-to-next-to-leading logarithmic soft-gluon resummation, Phys. Lett. B 710 (2012) 612 [arXiv:1111.5869] [INSPIRE].

[32] N. Kidonakis, Two-loop soft anomalous dimensions for single top quark associated production with a $W^{-}$or $H^{-}$, Phys. Rev. D 82 (2010) 054018 [arXiv:1005.4451] [InSPIRE].

[33] J.M. Campbell, R.K. Ellis and C. Williams, Vector boson pair production at the LHC, JHEP 07 (2011) 018 [arXiv:1105.0020] [INSPIRE].

[34] T. Sjöstrand, S. Mrenna and P.Z. Skands, A brief introduction to PYTHIA 8.1, Comput. Phys. Commun. 178 (2008) 852 [arXiv:0710.3820] [INSPIRE].

[35] J. Alwall, M. Herquet, F. Maltoni, O. Mattelaer and T. Stelzer, MadGraph 5: going beyond, JHEP 06 (2011) 128 [arXiv:1106.0522] [INSPIRE].

[36] J. Pumplin et al., New generation of parton distributions with uncertainties from global QCD analysis, JHEP 07 (2002) 012 [hep-ph/0201195] [INSPIRE].

[37] D. Stump et al., Inclusive jet production, parton distributions and the search for new physics, JHEP 10 (2003) 046 [hep-ph/0303013] [INSPIRE].

[38] S. Ovyn, X. Rouby and V. Lemaitre, DELPHES, a framework for fast simulation of a generic collider experiment, arXiv:0903.2225 [INSPIRE].

[39] CMS collaboration, G.L. Bayatian et al., CMS physics technical design report volume I: detector performance and software, CERN-LHCC-2006-001 (2006).

[40] M. Cacciari, G.P. Salam and G. Soyez, FastJet user manual, Eur. Phys. J. C 72 (2012) 1896 [arXiv: 1111.6097] [INSPIRE].

[41] T. Junk, Confidence level computation for combining searches with small statistics, Nucl. Instrum. Meth. A 434 (1999) 435 [hep-ex/9902006] [INSPIRE].

[42] A. Read, Modified frequentist analysis of search results (the CLs method), CERN-OPEN-2000-205 (2000).

[43] CMS collaboration, V. Khachatryan et al., Measurements of inclusive $W$ and $Z$ cross sections in pp collisions at $\sqrt{s}=7$ TeV, JHEP 01 (2011) 080 [arXiv:1012.2466] [INSPIRE].

[44] ATLAS collaboration, G. Aad et al., Measurement of the inclusive $W^{ \pm}$and $Z / \gamma$ cross sections in the electron and muon decay channels in pp collisions at $\sqrt{s}=7$ TeV with the ATLAS detector, Phys. Rev. D 85 (2012) 072004 [arXiv:1109.5141] [INSPIRE]. 